ISSN 1112-9867

Available online at

http://www.jfas.info

\title{
CONTRIBUTION OF GRAVIMETRY TO THE HYDROGEOLOGICAL RECOGNITION OF THE AREA OF WADI RIGH -OUARGLA
}

\author{
O. Melouah*, A. Zeddouri \\ Université Kasdi Merbah, Laboratoire des réservoirs souterrains Pétroliers, Gaziers et \\ Aquifères, BP 511, Route Ghardaïa, Ouargla, Algérie
}

Received: 18 September 2016 / Accepted: 21 December 2016 / Published online: 01 January 2017

\begin{abstract}
Gravimetry is a geophysical methods often used in oil exploration and mining research but rarely in the hydrogeological field, the aim of this work is to exploit the gravimetric data to develop a structural model of the region of Oued Righ - Ouargla has less than 1,000 m depth and perform a litho-stratigraphic modeling of subsurface formations to assess the ability of a karst formation of the terminal complex water table (Eocene Senonian).

Multiple filtrations and signal processing techniques for gravimetric datas were operated such as the Euler Déconvolution, the analytical signal and the horizontal gradient.

The results were used to evaluate the potential of karstification in the area and to model the phenomenon; the subsurface structural mapping has also led to the relationship between the well productivity and network of faults and fractures.
\end{abstract}

Key words: Euler deconvolution, analytic signal, faults, karst

Author Correspondence, e-mail: oualid411@yahoo.fr

doi: http://dx.doi.org/10.4314/jfas.v9i1.6

\section{INTRODUCTION}

Dans la région d'Ouargla le complexe terminale englobe plusieurs nappes parmi la quelle la nappe sénonienne, celle-ci percole à travers les formations carbonates et lagunaires, 
l'écoulement de la nappe s'effectue du Sud-ouest vers le Nord Est, l'utilisation de la gravimétrie va permettre d'élaborer un modèle structural de la région afin de mieux interpréter la distribution de la ressource en eau [1].

Dans sa plus grande partie le CT est constitué de nappes carbonatées (Eocène, sénonien, turonien) certains endroits de la nappe se caractérisent par des débits importants, à travers ce travaille on essai de mettre en évidence l'effet de la karstification et la fracturation sur les potentialités hydrogéologiques de l'aquifère du CT.

L'étude des karsts contribue à la bonne gestion des ressources en eaux superficielles et profondes .divers approches dans le monde ont traité cette problématique, [2]; [3]; [4]; [5].

La Préservation des ressources profondes implique forcément la connaissance de la morphologie des karsts, l'Exokarst est la partie superficielle de celui-ci il assure le transfert et la canalisation des eaux collectées de la surface vers les réservoirs profonds ou Endokarsts, $[6] ;[7] ;[8]$.

Peu d'études ont traité La karstification dans le Sahara Algérien ce travaille met en évidence le phénomène dans la nappe sénonienne par le biais de la modélisation géologique et les techniques modernes utilisées en gravimétrie.

\subsection{Contexte Géologique et Hydrogéologique}

La région d'Ouargla, au Nord-Est du Sahara algérien, est limitée au Nord par la Sebkha Sefioune, au Sud par les sites de Hassi Messaoud et Gassi Touil, à l'Ouest par la Dorsale du Mzab et à l'Est par Haoud El Hamra et le grand Erg Oriental. La cuvette d'Ouargla est creusée dans les sédiments de la vallée fossile de l'Oued Mya, constituées essentiellement de grès de calcaire Mio-pliocène et d'alluvions Quaternaires.

En profondeur l'Eocène et le Sénonien marin sont carbonatés alors que le Sénonien lagunaire est constitué d'anhydrite, halite, gypse et quelque trace de marne et d'argile. Le Turonien est carbonaté et parfois dolomitique.

$\mathrm{Du}$ point de vue potentialité Hydrogéologique la région abrite deux ensemble Hydrogéologiques le complexe terminale CT et le continental intercalaire CI 
La nappe du complexe terminale est constituée des eaux de la nappe des sables du Mio-pliocène, des nappe carbonatées Sénoniennes, Turoniennes.
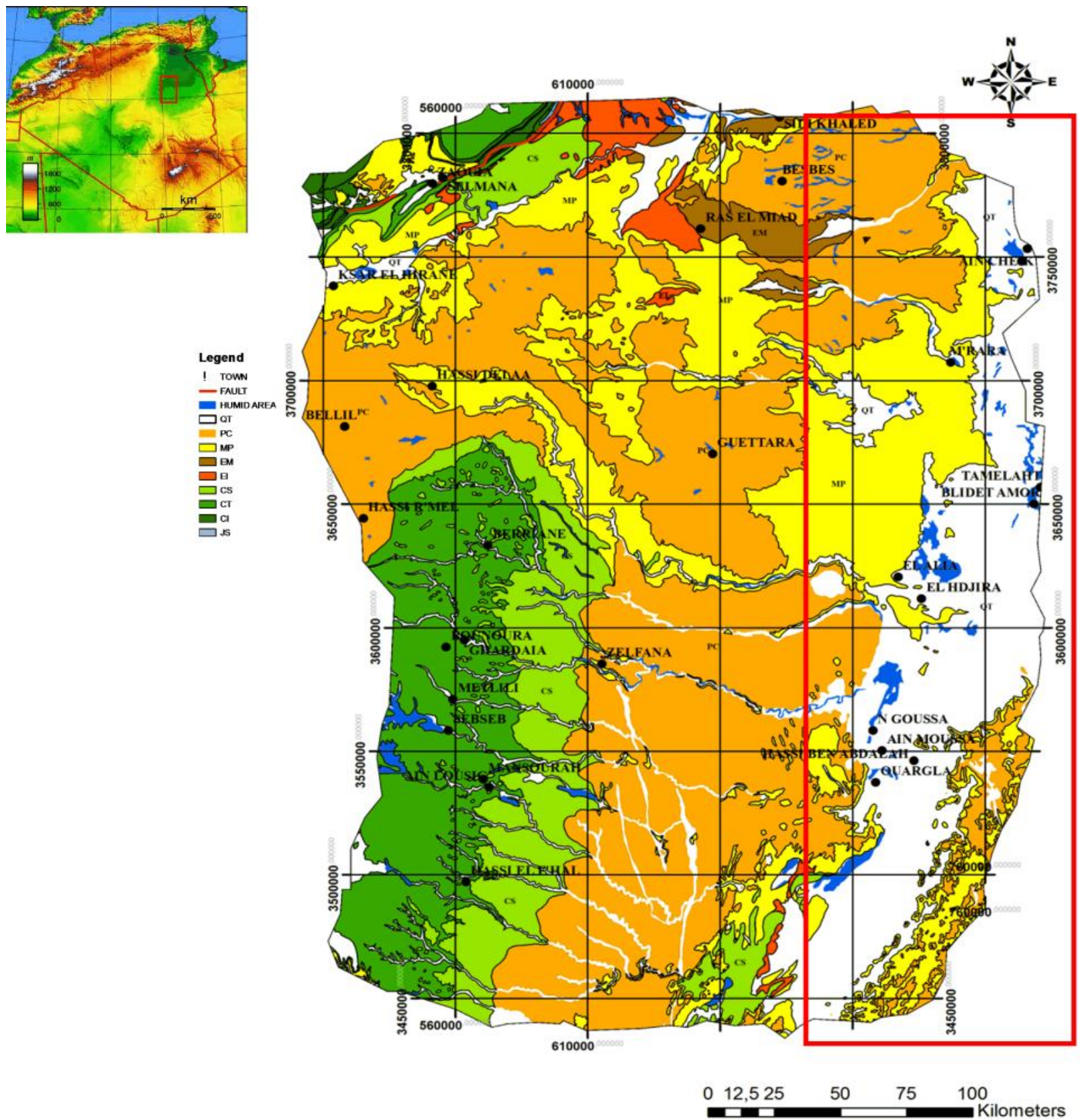

Fig.1. Carte géologique du secteur d'étude.

QT : Quaternaire, PC : Pliocène continental, MP : Mio-pliocène, EM :Eocène moyen, EI : Eocène inferieur, CS : Crétacé supérieur, CT: Crétacé moyen (turonien), CI : Crétacé inferieur, JS : jurassique supérieur 


\section{MATERIELLES ET METHODES}

La méthodologie proposée pour la mise en évidence des karsts au Nord du Sahara Algérien se résume en deux étapes

-1) L'utilisation des méthodes gravimétriques et des techniques modernes du traitement du signal

-2) La modélisation et la cartographie des données géologiques obtenus à partir des logs de forages et des essais de pompages réalisés au paravent.

\section{1) La Gravimétrie :}

L'anomalie gravimétrique de Bouguer est l'écart entre le champ de pesanteur terrestre mesurée et corrigée et le champ de pesanteur théorique.

Les données gravimétriques obtenus proviennent de la base de données du BGI (Bureau Gravimétrique international), l'anomalie de Bouguer et calculer par une densité de 2.670 $\mathrm{g} / \mathrm{cm}^{3}$, Ces données sont issues des compagnes d'acquisitions terrestres et aéroportés.

Les informations obtenues à partir de la carte des anomalies de Bouguer se limitent à la distribution des hétérogénistes gravimétriques due aux contrastes des densités entre les matériaux géologiques.

Afin de mieux exploités ces résultats (obtention des linéaments tectoniques) un traitement spécifique par filtration du signal gravimétrique s'avère indispensable.

La première étape consiste à séparer les anomalies gravimétriques régionales et résiduelles , par l'application de la technique des polynômes.

La deuxième étape consiste à l'application des techniques d'interprétations tell que la Déconvolution d'Euler et le Gradient Horizontal et le signal analytique

\subsection{1) Déconvolution d'Euler}

La Déconvolution d'Euler [9]; [10] est une technique utilisée pour la localisation des sources gravimétriques et magnétiques, elle est basée sur la résolution de L'Equation d'homogénéité d'Euler :

$$
\left[\left(x-x_{0}\right) \partial \mathrm{T} / \partial x\right]+\left[\left(y-y_{0}\right) \partial \mathrm{T} / \partial y\right]+\left[\left(z-z_{0}\right) \partial \mathrm{T} / \partial z\right]=N(B-T)
$$

Où $\left(\boldsymbol{x}_{0}, \boldsymbol{y}_{0}, z_{0}\right)$ sont les positions des sources gravimétriques et magnétiques détectées à partir du champ total $\mathbf{T}$ aux positions $(\boldsymbol{x}, \boldsymbol{y}, \boldsymbol{z})$, le champ total a une valeur régional $\boldsymbol{B}$, le degré 
d'homogénéité $\boldsymbol{N}$ peut être interprété étant que Indice Structural SI [10].

La résolution du système d'équation dépend de l'indice structural choisie, un mauvais choix peut conduire à des Profondeurs exagérées ou sous-estimées [10],

Plusieurs approches sont proposées pour déterminer la valeur de ce paramètre, En gravimétrie $N$ a des valeurs appropriées à chaque type de structure ,Pour les dykes et les contacts $N=0$, pour les pipes $N=1$, pour les objets sphériques $N=2$ [11], d'autres auteurs donnent des valeurs différentes de l'indice structurale [9], Reid et al (1990) estime un indice structural égale à 1 pour les filons minces, les dykes et les failles à faibles rejets vertical, un indice $\mathrm{N}=0$ pour les failles à grands rejets et un indice de 0.5 pour les cas intermédiaires [10] .

La valeur correcte de ce paramètre peut ce traduire par la dispersion la moins importante des solutions obtenues [9] ; [12] ; [13]. [14], dans ce travaille plusieurs indices structuraux on était testés $(\mathbf{N}=\mathbf{0}, \mathbf{N}=\mathbf{0 . 5}, \mathbf{N}=\mathbf{1})$, les solutions les plus appropriées et qui vérifient ces conditions sont obtenues par indice structural 0

Cette approche laisse une large place au facteur humain, avec des risques d'erreurs dans le choix de l'indice structural. D'autre travaux ont été réalisés dans ce sens afin de réduire l'intervention du paramètre humain, Thompson (1982), a développé un algorithme mathématique (EULDPH) capable de déterminer la profondeur des sources magnétiques et gravimétriques sans l'introduction des informations concernant la géométrie de la source [9] , Barbosa (1999), propose un nouveau critère mathématique pour le choix de l'indice structural basé sur la corrélation entre le champ total des anomalies et les estimations d'un niveau de base inconnue [15], ces estimations sont obtenues pour chaque position de la fenêtre des données se déplaçant le long d'un profil observé et pour plusieurs valeurs indicatives pour l'indice structural, la corrélation la moins significatif représente le meilleur choix de cet indice

\subsection{2) Le Gradient Horizontal :}

Le gradient horizontal est une technique basée sur le calcule des dérivées selon $\boldsymbol{x}, \boldsymbol{y}$ d'un champ de potentiel, la méthode a était utilisé pour la localisation des corps à fort contraste gravimétrique et magnétique [16], l'amplitude d'un gradient horizontal est exprimé par :

$$
G H=\left[(\partial g / \partial x)^{2}+(\partial g / \partial y)^{2}\right]^{1 / 2}
$$


Où $(\partial g / \partial x)$ et $(\partial g / \partial y)$ sont les dérivées horizontales selon $\boldsymbol{x}$ et $\boldsymbol{y}$ du champ de gravité.

Les maximas du gradient horizontal calculé selon la méthode de Blakley et Simpson (1985) [17], permettent la localisation des contacts géologiques liés à la présence de failles et discontinuités géologiques [16] ; [18].

Le pendage des contacts géologiques peut être déterminé par le prolongement vers le Haut de la carte du gradient horizontal à divers altitude [19]

Les niveaux de prolongement les plus élevés correspondent au structures géologiques les plus profondes [18] ; [20].

\subsection{3) Signal Analytique :}

Selon Roest (1992) et Bournas (2003) [21] ; [14] ,le signal analytique peut être défini par :

$$
A S(x, y)=[(\partial g / \partial x) \hat{x}+(\partial g / \partial y) \hat{y}-i(\partial g / \partial z) \hat{z}]
$$

Où g représente l'intensité du champ de potentiel, $\hat{x}, \hat{y}, \hat{z}$ Sont les vecteurs unitaires selon les directions $\quad x, y, z, i$ est un nombre complexe.

Les maximas du signal analytique permettent de délimiter les anomalies du champ de potentiel.

\section{2) La modélisation géologique :}

Plus de 130 forages ont était exploité afin de réaliser un modèle stratigraphique dans la région d'Ouargla, Hassi Messoud et Hadjira, la base de donnés collectés a servie à modélisé l'extension des formations susceptible d'être karstique, le degré de confiance du model est de $95 \%$, plusieurs filtre ont était appliqués à la surface et à la base du modèle afin de quantifier correctement les volumes occupés par les formations, l'algorithme utilisés pour l'interpolation est le krigage.

\section{RESULTATS ET DISCUSSION}

L'application d'un filtre polynomial d'ordre 1 sur 1360 points de mesures gravimétriques à permis d'obtenir la carte des anomalies résiduelles représentés dans la figure 2a, le maillage choisie est de $3 \mathrm{~km}^{2}$ entre chaque point de mesure ,la distribution des densités est homogène, 
au Nord de Meghaier une structure à anomalie négative de -13 mgal est causée par le bassin de chott Melghig, entre Djamaa, M'rara et N'Goussa une structure à anomalie positive de l'ordre de 3 à 14 mgal occupe le centre, généré par la structure en Horst de Djamaa, Touggourt, Hassi Messaoud ,au sud de Blidet Amor deux failles sont observées .

En allant vers Ouargla, Hassi Messoud le signal gravimétrique est inversé témoignant de la présence de bassin sédimentaire épais.

Sur les cartes représentées dans les figures $2 b, 2 d$ les maximas du signal analytique et du gradient horizontal permettent de localiser plusieurs anomalies rectilignes orientées vers la direction N30-40 et qui représente probablement les failles majeures dans la région.

Ces déformations sont les résultats des phases: autrichienne (compressive de direction Est-Ouest), Eocène (compressive N-S), Miocène (compressive N-S, NW-SE) et Villafranchienne (compressive N-S)

L'étude de ces orientations va permettre de déduire la relation entre les karst et la répartition des linéaments tectoniques d'une part, d'autre part a confirmer la présence d'une continuité hydrogéologique à travers les nappes du complexe terminal (Nappes du Moi-Pliocène, Eocène et Sénonien carbonatés) .

La Déconvolution d'Euler appliquée aux données gravimétriques à Permis d'obtenir divers solutions.

En littérature afin de déterminé les contacts géologiques et les linéaments le meilleur choix serait d'utiliser l'indice structural $\mathrm{N}=0$ [18] ; [19]; [11], l'ensemble des solutions obtenues après la résolution de l'équation d'homogénéité sont représentés dans la fig.2 C.

Le choix des paramètres de la Déconvolution d'Euler $(\mathrm{N}=0$, largeur fenêtre de $10 \times 10$, erreur relatif de 15\%) à permis de déterminer la profondeur des sources gravimétriques (fig.2 C), et de classer les linéaments selon les directions prédominantes N 30-60, N120-140.

Les linéaments sont situés à des profondeurs variables entre 200-400 m, 400-600 $\mathrm{m}, 600-800 \mathrm{~m}$.

Les formations affectées sont généralement d'âge Cénozoïque, Les orientations obtenues après La combinaison des résultats de la Déconvolution d'Euler avec Les Maximas du gradient Horizontal et le signal analytique sont $\mathrm{N} 20^{\circ}-50^{\circ}, \mathrm{N} 120^{\circ}-150^{\circ}$ (Fig. 5.a). 
a

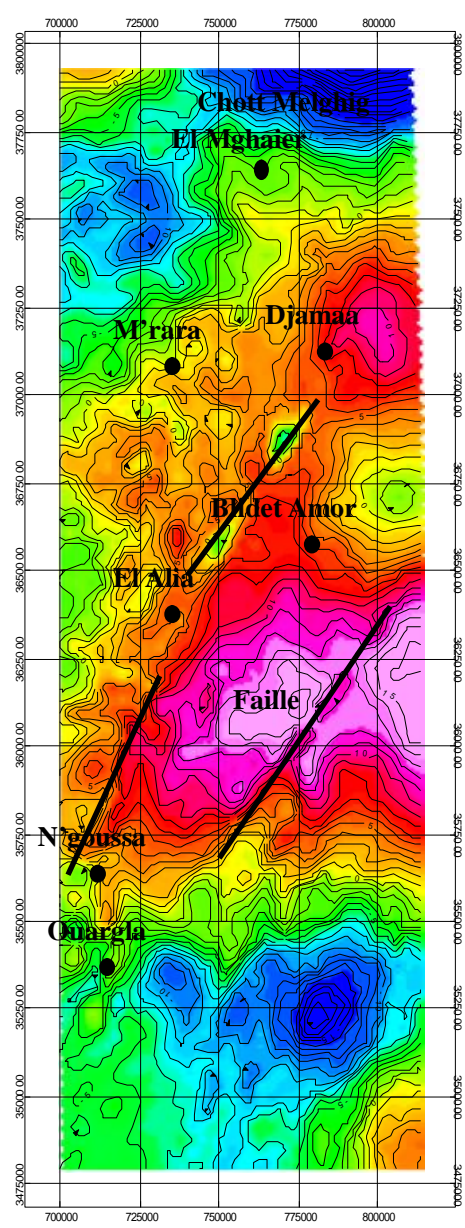

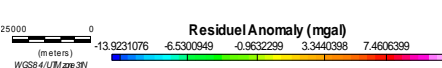

b
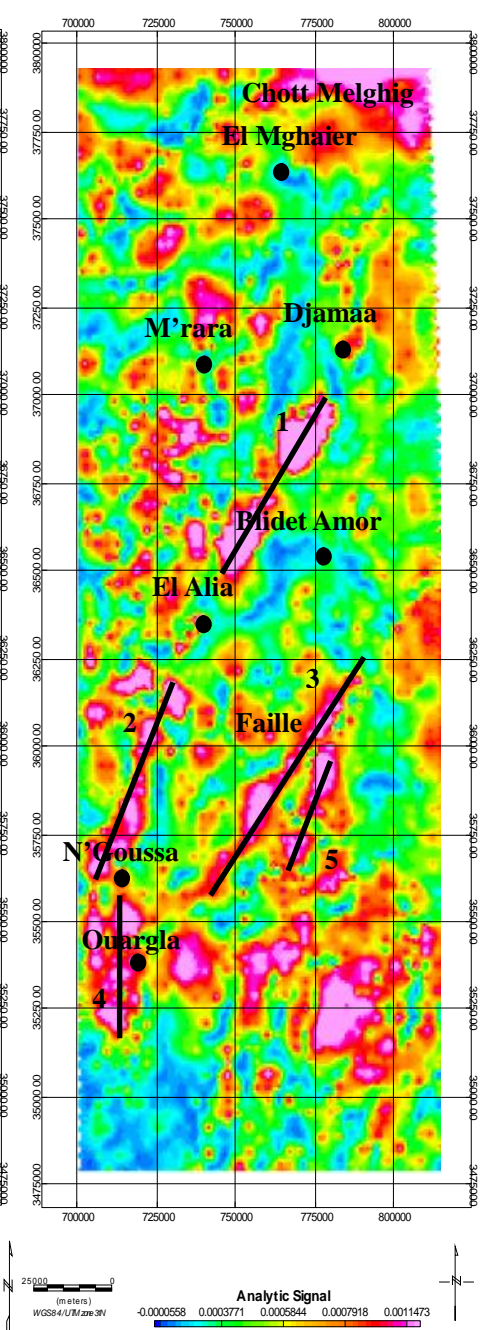

$\mathrm{C}$

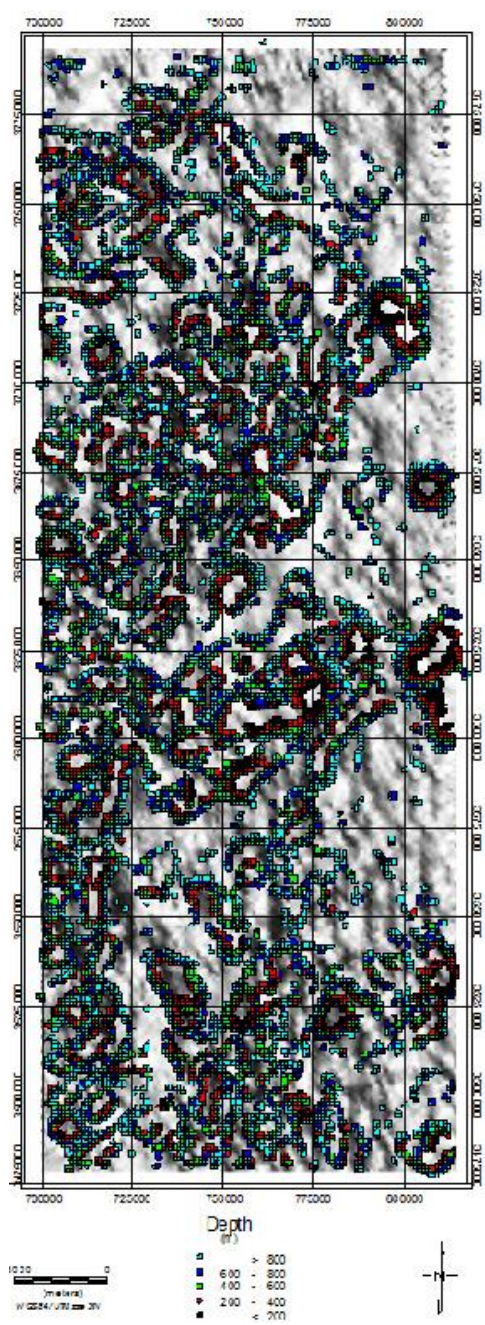

$\mathrm{D}$

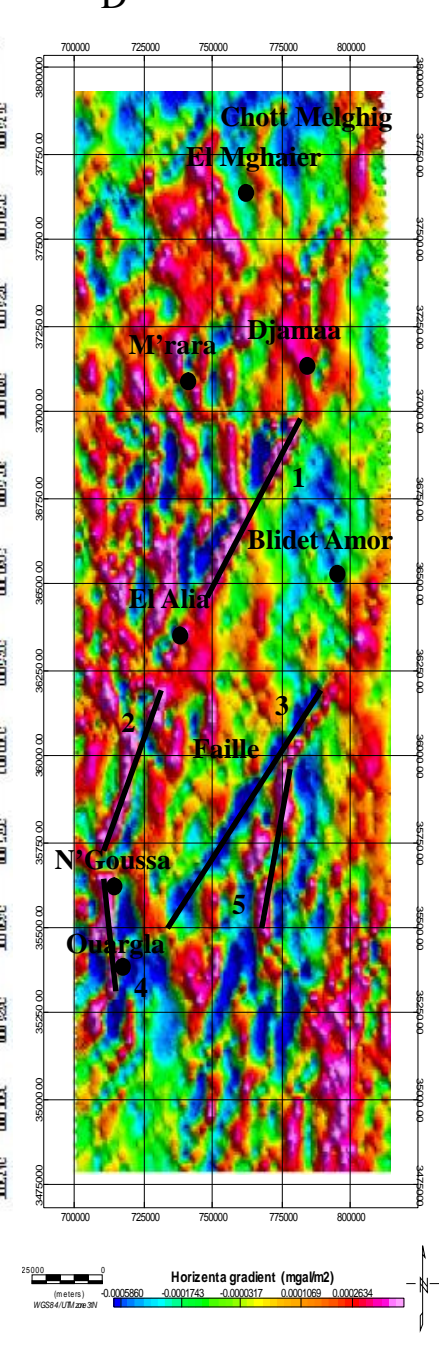

$\sqrt{20}$

Fig.2. a) carte des anomalies résiduelles polynôme d'ordre 1, b) résultats du signal analytique,

C) Localisation des solutions de la déconvolution d'Euler en fonction de la profondeur des sources gravimétriques, D) résultats du gradient horizontal.

Le terrain choisie pour cette étude présente une aptitude à la karstification, la coupe hydrogéologique dans la figure 3 est orientée vers la direction N-S, le Mio-Pliocène est constitué essentiellement de formation perméable et semi perméable (sable ,argile et argile sableuse ) le volume total occupé par ces formations dans la région d'Ouargla -Hassi Messaoud- Hadjira est estimé à environ $6,22.10^{12} \mathrm{~m}^{3}$,ces résultats sont obtenues à partir de l'élaboration d'un modèle stratigraphique couvrant 130 forages .

à partir de Djamaa et jusqu'à la bordure Sud de l'atlas saharien le volume des sédiments 
Mio-Plio-Quaternaires augmente considérablement, le substrat est purement argileux (Eocène .moyen) réduisant les possibilités d'un contact probable entre les nappes du Plio-Quaternaire - l'Eocène inferieur et du Sénonien carbonatés ,le sillon de Melghig représente la zone d'accumulation des eaux du Mio-Pliocène, la profondeur des forages peut dépasser les 500m, l'épaisseur du substrat argileux entre Ouargla-Touggourt diminue considérablement (figure 3), les calcaires phosphatés de Eocène sont dispersés de part et d'autre et ne constituent pas une formation géologique continue et à vaste étendue le volume est estimé à 2,16.10 $12 \mathrm{~m}^{3}$ (figure $4 \mathrm{C}$ ). Le Sénonien est subdivisé en trois grands ensembles : le Sénonien carbonaté (volume estimé $=5,9.10^{12} \mathrm{~m}^{3}$ ), le Sénonien Anhydritique (volume estimé $=8,44 \cdot 10^{12} \mathrm{~m}^{3}$ ) et le Sénonien salifère (volume estimé $=4,39 \cdot 10^{12} \mathrm{~m}^{3}$ ). La présence de ce dernier est limitée à la partie Nord Est du Sahara Algérien.

Ces données préliminaires permettent de dire que la zone peut abriter des karsts vue le volume important des formations carbonatées et solubles, durant plusieurs travaux de forage des pertes énormes en boue sont enregistrés surtout entre les formations de l'Eocène et du Sénonien (carbonates et Anhydritique).

Ces pertes sont des paléo-karsts couverts par les formations superficielles et colmatés par les dépôts, souvent dans l'hydrogéologie de la région en sous-estime les potentialités des nappe de L'Eocène et du Sénonien carbonaté vue les faibles débits fournies, mais on se pose jamais la question sur leur entité hydrogéologique : sont elles des nappes karstiques colmatés ou des nappes fissurales?

Le modèle stratigraphique conçue avec un degré de confiance estimé à $95 \%$ a permis de calculer le volume du karst dans la région (figure 4 D), la première étape de la karstification est la disponibilité d'un réseau hydrographique dense et actif assurant le ruissellement des eaux vers les profondeurs, durant la phase Holocène le Sahara était une zone humide et marécageuse l'infiltration des eaux vers les carbonates peut être assurer par les formations superficielles du Mio-Pliocène ,la deuxième étape est la présence d'un réseau de fracture et de faille pour faciliter l'érosion chimio-mécaniques de ces roches, entre la phase autrichienne et villafranchienne plusieurs événements tectoniques ont eu lieu favorisant l'hypothèse d'un réseau de fracture ouvert dans les carbonates. Cela se confirme par les résultats obtenues par 
la gravimétrie, la figure 5a représente la cartographie structural de la région d'Oued Righ Ouargla à moins de $1000 \mathrm{~m}$ de profondeur. Ce document est indispensable à l'interprétation des phénomènes hydrogéologiques présents dans la région (apparition et alimentation des chotts).

Tableau 1 : volume de formations estimées après modélisation géologique

\begin{tabular}{lc}
\hline Formation & Volume estimé $\left(\mathrm{m}^{3}\right)$ \\
\hline Moi-pliocène & 6222713222389 \\
Eocène & 2162575480892 \\
Karst & 888717023479 \\
Sénonien carbonaté & 5907553325015 \\
Senonien Anhydritique & 8442707570423 \\
Senonien Salifère & 4395225353872 \\
Volume des formations solubles $\left(\mathrm{m}^{3}\right)$ & 20908061730202 \\
$\%$ occupé par le karst dans les formations solubles & $4.25 \%$ \\
\hline
\end{tabular}

$\mathbf{N}$

S

Saharian Atlas Erg Oriental

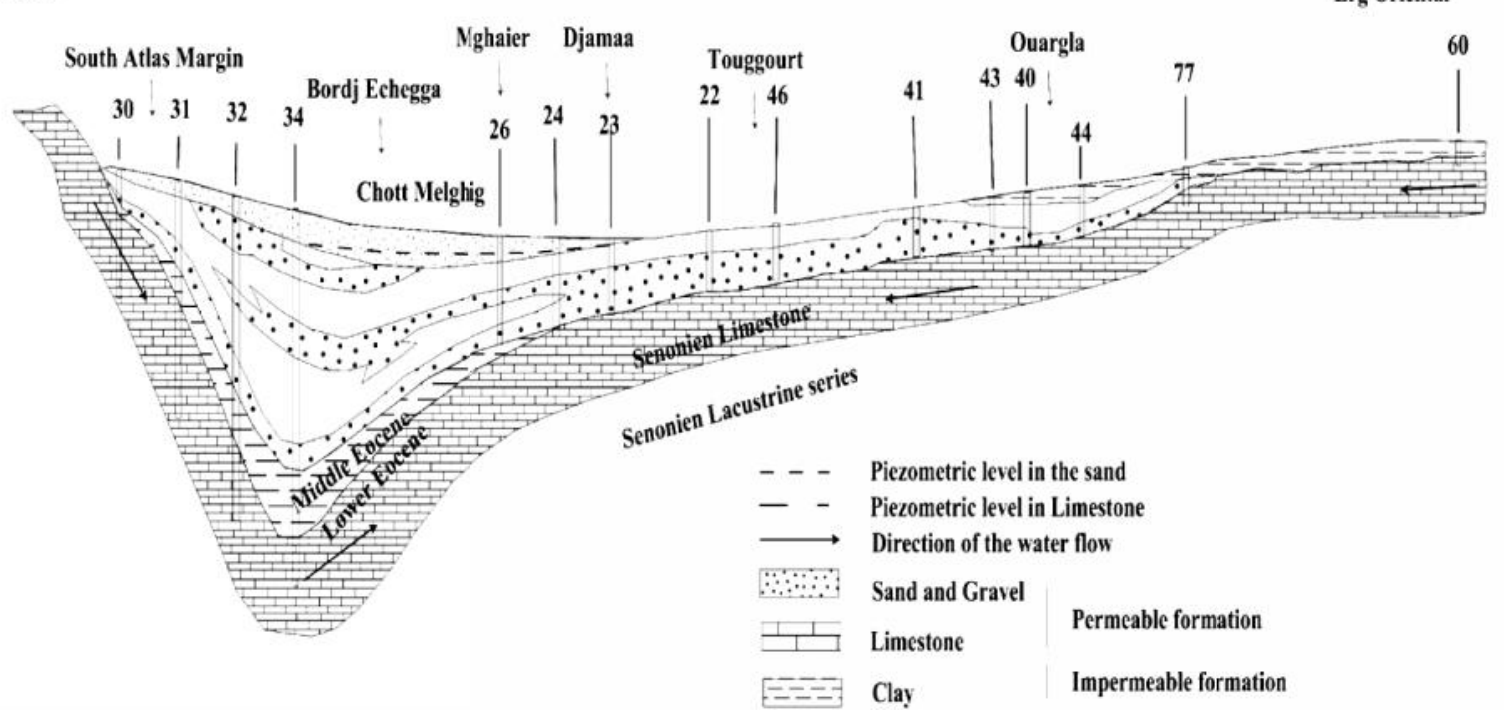

Fig.3. Coupe hydrogéologique de la région d'Oued Righ - Ouargla 

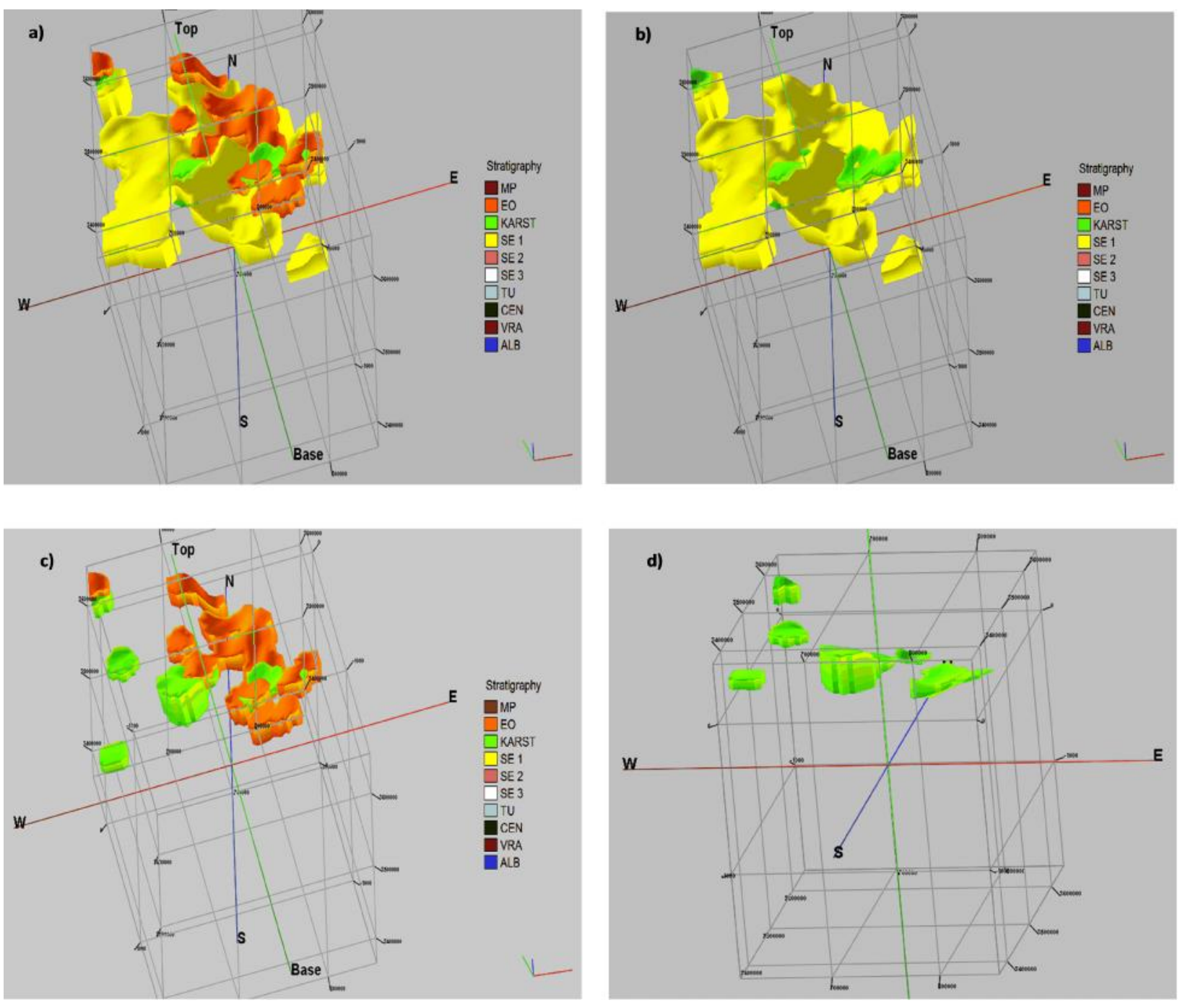

Fig.4. Modèle stratigraphique en 3D de la région d'Ouargla, Hassi Messoud et Hedjira MP : Mio-pliocène, EO : Eocène, SE1 : Senonien carbonaté, SE2 : Senonien Anhydritique, SE3 : Sénonien Salifère, TU : Turonien, CEN : Cénomanien, VRA : vraconien, ALB : Albien.

Le volume occupé par le karst représente 4.25\% (tableau 1) du volume total des formations solubles dans le région, l'analyse des logs de forage montre l'absence de karst dans la région de Touggourt et ses environs, contrairement à Berkaoui et Hassi Messaoud (figure 4 a,b,c,d). La figure 5a résulte de la combinaison des techniques gravimétriques (Déconvolution d'Euler, Signal analytique et le gradient horizontal), les lignes rouges représentes des failles majeurs orientées entre $\mathrm{N} 20-40^{\circ}$ ces évenements correspondent aux phases situées entre l'autrichienne et la villafranchienne ,les élements representés en traits noirs correspondent à des failles de 
second ordre ou des diaclases .

La carte representé dans La figure 5b resume les débits obtenus pour plus de 100 forages exploitants la nappe du comlpexe terminale dans la vallée d'Oued righ .

Les débits les plus importants sont estimés à plus de 60 1/s, les plus faibles sont causés par l'effet des barrières géologiques ou des colmatages argileux, l'analyse de la figure 5b évoque la question suivante : y 'a-t-il une relation entre la productivité de la nappe du complexe terminal, la localisation du réseau de failles et la possibilité d'un drainage karstique dans la vallée d'Oued Righ ?

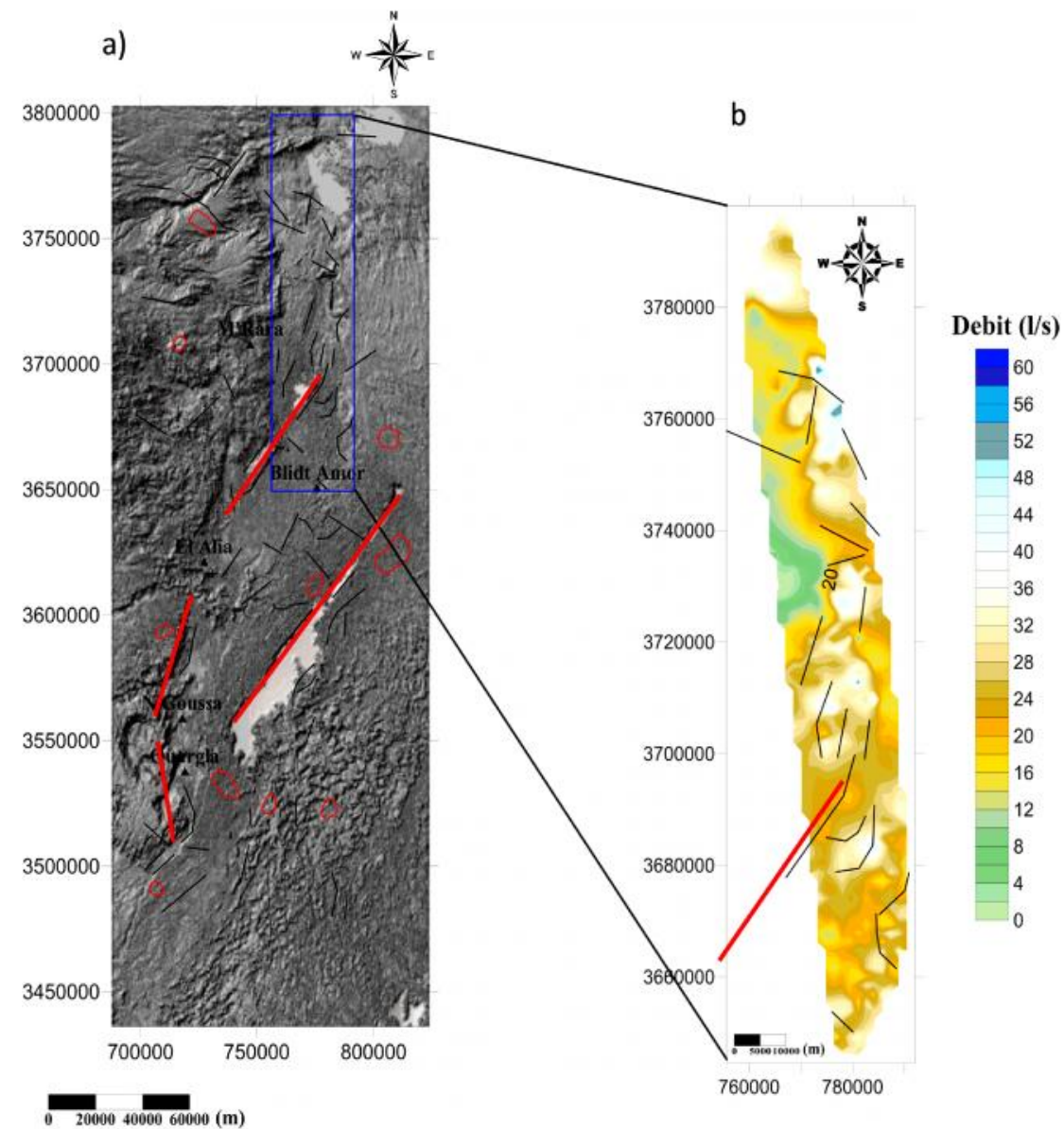

Fig.5.a) Carte structurale obtenu à partir de la combinaison des trois techniques gravimétriques, en noir les linéaments gravimétriques, en rouge les structures circulaires relatives aux diapirisme et aux remontés du socle, b) superposition de la carte des débits d'eau et la carte structurale 


\section{CONCLUSION}

Le travail présenté met en évidence l'efficacité de la gravimétrie dans le domaine de l'hydrogéologie, plusieurs failles majeurs de direction $\mathrm{N} 20-50^{\circ}$ sont identifiées .La modélisation géologique a permis d'avoir une idée sur les potentialités de la région, un total de $2,09.10^{13} \mathrm{~m}^{3}$ de matériaux solubles constituent les formations du complexe terminale .le karst occupe environ $4.25 \%$ de la totalité du potentiel soluble ,la productivité de la nappe du complexe terminal est en relation avec le réseau de fractures et des failles traversant la région .

La question qui se pose à travers ces travaux concerne la fonctionnalité de ce karst : s'agit il donc d'un karst fonctionnel actuellement ou d'un paleokarst colmaté est qui n'as pas continué son évolution vue les changements climatiques au cours des temps géologiques?

\section{REMERCIMENTS}

Les auteurs tiennent à remercié le bureau gravimétrique international (BGI, Observatoire Midi-Pyrénées 14, Avenue Edouard Belin 31400 Toulouse, France) pour la facilité d'accès au données, et le laboratoire des Réservoirs Souterrains Pétroliers, Gaziers et Aquifères (LRSPGA, université Kasdi-Merbah Ouargla, Algérie) pour les efforts fournis par son personnelles durant la réalisation de ces travaux.

\section{REFERENCES}

[1] Setianingsiha, Efendib R, Abdul Kadira W G, Santosoa D, Idham Abdullahc C, Alawiyah S. Procedia Environmental Sciences. 17, 2013, 248 - 255, doi: 10.1016/j.proenv.2013.02.035. [2] Papadopoulou-Vrynioti K, Bathrellos G D, Skilodimou H D, Kaviris G, Makropoulos K. Engineering Geology. 158, 2013, 77-88, http://dx.doi.org/10.1016/j.enggeo.2013.02.009.

[3] Martínez-Moreno F J, Pedrera A, Ruano P, Galindo-Zaldívar J, Martos-Rosillo S, González-Castillo L, Sánchez-Úbeda J P, Marín-Lechado C. Engineering Geology.162 2013, 67-78, http://dx.doi.org/10.1016/j.enggeo.2013.05.008.

[4] Benac C, Juračić M, Matičec D , Ružić I, Pikelj K. Geomorphology. 184, 2013, 64-73, DOI: 10.1016/j.geomorph.2012.11.016 
[5] Alexakis D, Tsakiris G .Desalination and Water Treatment .16 (1-3), 2010, 229-237, DOI: 10.5004/dwt.2010.1065

[6] Waltham A C and Fookes P G .Engineering classification of karst ground conditions. Speleogenesis and Evolution of Karst Aquifers ., 2005, 3 (1): 1-20.

[7] Waele J D, Plan L, Audra P. Geomorphology, 106, 2009, 1-8, Doi:10.1016/j.geomorph.2008.09.023.

[8] Emblanch C, Zuppi, G M, Mudry J, Blavoux B, Batiot C. Journal of Hydrology, 279(1),2003 , 262-274, DOI: 10.1016/S0022-1694(03)00180-X

[9] Thompson D T. Geophysics, 47 (1), 1982, 31-37, DOI: 10.1190/1.1441278

[10] Reid A B, Allsop J M, Grancer H, Millett A J and Somerton I W. Geophysics , 55 (1), 1990, 80-91, Doi: 10.1190/1.1442774.

[11] Khalil M A, Santos F M, Farzamian M. Journal of applied Geophysics, 103, 2014, 104-113, Doi: 10.1016/j.jappgeo.2014.01.012.

[12] Feng-e Z, Ji-xiang Q, Yao-ru L, Sheng Z, Mi-ying Y. Procedia Earth and Planetary Science, 7, 2013 , 944 - 947, Doi:10.1016/j.proeps.2013.03.148.

[13] Barbosa V C F, Silva J B C, Medeiros W E. Journal of Applied Geophysics, 43 (1), 2000, 55-68, DOI: 10.1016/S0926-9851(99)00047-6.

[14] Bournas.N , Galdeano A, Hamoudi M, Baker H. Journal of African Earth Sciences, 37 (3-4) ,2003, 191-205, DOI: 10.1016/j.jafrearsci.2002.12.001.

[15] Barbosa V CF, Silva J B C, Medeiros W E. Geophysics, 64(1),1999, 48-60, DOI: $10.1190 / 1.1444529$.

[16] Cordell L, Grauch, V J S. Mapping basement magnetization zones from aeromagnetic data. In: 52nd Ann. Int. Meet. Soc. Explor. Geophys. Dallas. The San Juan Basin, New Mexico, 1982, pp. 246-247 (abstracts and biographies).

[17] Blakely R J, Simpson R W. Approximating edges of source bodies from magnetic and gravity anomalies. Geophysics., 1986, 51(7), 1494-1498.

[18] Khettache D, Keating P, Mili E M, Chennouf T, Andrieux P, Milhi A. C.R.Géosciences, 336 (16),2004,1427 -1432 .Doi : 10.1016/j.crte.2004.09.012.

[19] El-Gout R, Khattach D, Houari M R, Kaufmann O, Aqil H. Journal of African Earth 
Sciences, 58(2),2010, 255-271, DOI: 10.1016/j.jafrearsci.2010.03.006

[20] Chennouf T,khattach D,milhi A,keating P. C.R.Geosciences, 339(6), 2007, 383-395, ·http://dx.doi.org/10.1016/j.crte.2007.03.006

[21] Roest W R, Arkani-Hamed J and Verhoef J, Geophysi. J. I, 109(3), 1992, 653-669, Doi: 10.1111/j.1365-246X.1992.tb00123.x

\section{How to cite this article:}

Melouah O, Zeddouri A. contribution of gravimetry to the hydrogeological recognition of the area of Wadi righ -ouargla. J. Fundam. Appl. Sci., 2017, 9(1), 74-88. 